\title{
Comparisons between non-alcoholic steatohepatitis and alcohol-related hepatocellular carcinoma
}

\author{
Rahul Kumar', Boon-Bee George Goh², Jia-Wen Kam, Pik-Eu Chang², and Chee-Kiat $\operatorname{Tan}^{2}$ \\ 'Department of Gastroenterology and Hepatology, Changi General Hospital; ${ }^{2}$ Department of Gastroenterology and Hepatology, Singa- \\ pore General Hospital, Singapore
}

\section{Graphical Abstract}

Non-alcoholic and alcoholic steatohepatitis both cause cirrhosis and liver cancer but differences between NASH-HCC and ASH-HCC are unknown.

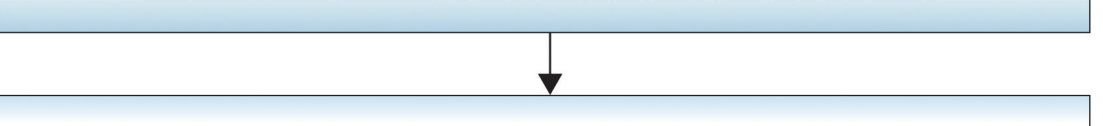

NASH-HCC patients were older at diagnosis, had significantly more metabolic risk factors and were less frequently diagnosed on surveillance compared to $\mathrm{ASH}-\mathrm{HCC}$.
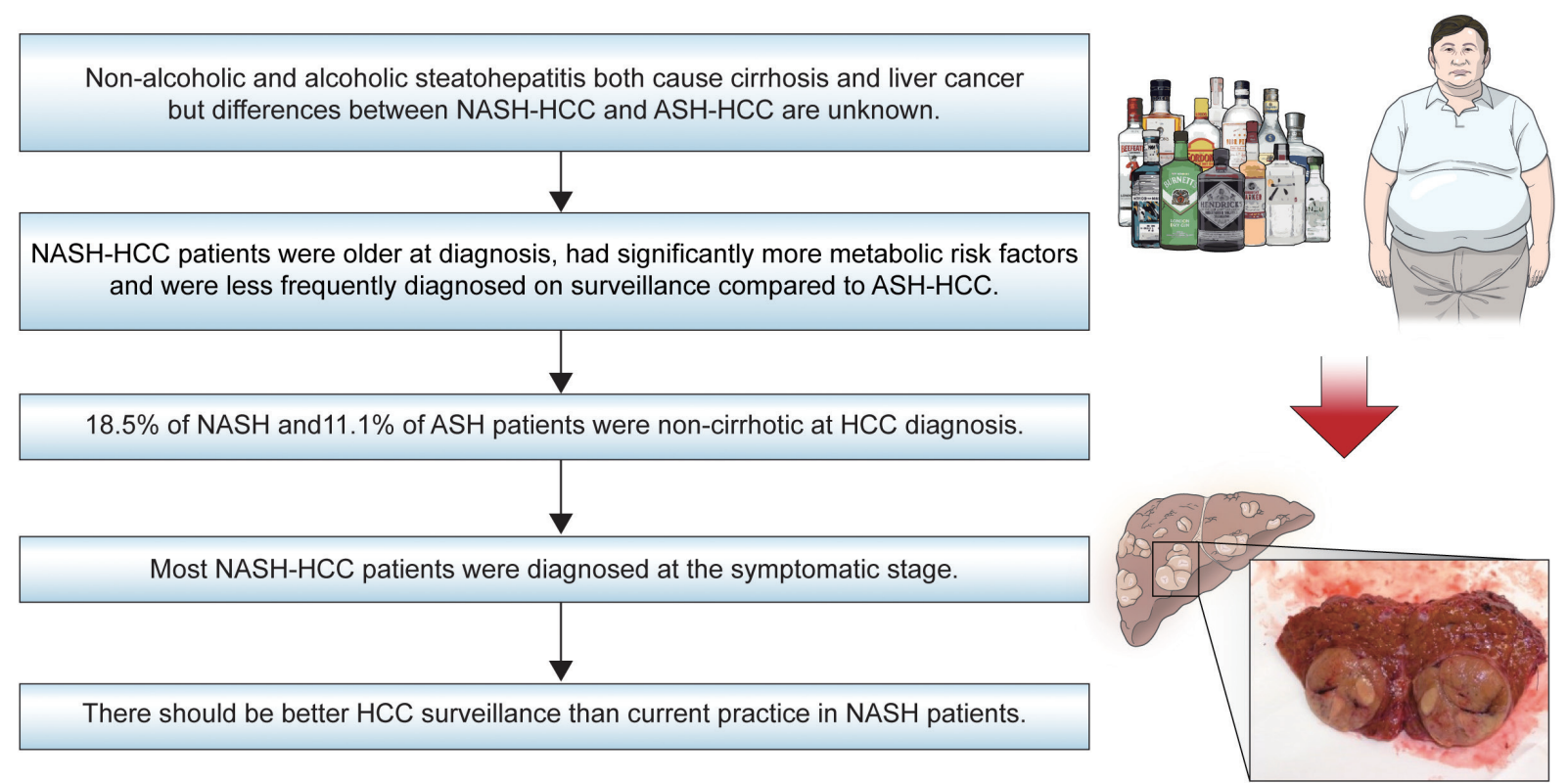

\section{Abbreviations:}

AFP, alpha-fetoprotein; Alb, albumin; ALD, alcoholic liver disease; ALP, alkaline phosphatase; ALT, aspartate transaminase; AST, aspartate aminotransferase: BCLC, Barcelona Clinic Liver Cancer; BMI, body mass index; CT, computerized tomography; CTP, Child-Turcotte-Pugh; DM, diabetes mellitus; HBV, hepatits B virus; $\mathrm{HCC}$, hepatocellular carcinoma; $\mathrm{HCV}$, hepatitis $\mathrm{C}$ virus; $\mathrm{MELD}$, model for end-stage liver disease; MRI, magnetic resonance imaging; NAFLD, non-alcoholic fatty liver disease; NASH, non-alcoholic steatohepatitis; OS, overall survival; PTEN, phosphatase and tensin homolog; Tbil, total bilirubin; TNM, Tumor lymph Node Metastasis

\section{Corresponding author : Chee-Kiat Tan}

Department of Gastroenterology and Hepatology, Singapore Genera Hospital, 20 College Road 169856, Singapore

Tel: +65-62223322

E-mail: tan.chee.kiat@singhealth.com.sg https://orcid.org/0000-0001-6992-2319 
Background/Aims: Non-alcoholic liver disease and alcoholic liver disease begin as simple steatosis that may progress to steatohepatitis and ensuing liver-related complications such as cirrhosis and hepatocellular carcinoma (HCC). We explored differences in characteristics between non-alcoholic steatohepatitis (NASH) and alcoholic steatohepatitisrelated (ASH) HCC.

Methods: NASH and ASH patients were identified from our department's prospective HCC database. A total of 54 and 45 patients met predefined inclusion and exclusion criteria for the NASH-HCC and ASH-HCC groups, respectively. Clinical, biochemical and tumor characteristics were studied.

Results: NASH-HCC patients were older compared to ASH-HCC patients ( $72 \pm 9$ vs. $66 \pm 9$ years, $P<0.001)$ and less male predominant (65\% vs. $98 \%, P<0.001)$. Prevalence of diabetes mellitus ( $78 \%$ vs. $36 \%, P<0.001)$ and hypertension ( $80 \%$ vs. $58 \%, P<0.001$ ) were significantly higher in the NASH-HCC group. Liver function tests and Child-Pugh scores were similar. There were no differences in alpha-fetoprotein level, lesions found at diagnosis (unifocal/multifocal) or prevalence of portal vein invasion. In both groups, almost half of the patients were in TNM stage 4 at the time of diagnosis and more than $50 \%$ of patients were not suitable for any therapy. Median survival in the NASH-HCC and ASH-HCC groups were 13 and 7 months respectively $(P=0.113)$.

Conclusions: Despite significant differences in demography of the NASH-HCC and ASH-HCC groups, liver and tumor characteristics were comparable. Most patients were diagnosed late and were not amenable to curative or locoregional therapies. Better characterization of patients with NASH and ASH at risk of HCC is necessary to optimize screening, surveillance, and management strategies. (Clin Mol Hepatol 2020;26:196-208)

Keywords: Carcinoma, Hepatocellular; Non-alcoholic fatty liver disease; Fatty liver, Alcoholic; Survival

\footnotetext{
Study Highlights

1. NASH-HCC patients were older at diagnosis, had significantly more metabolic risk factors and were diagnosed less frequently on surveillance as compared to ASH-HCC.

2. $18.5 \%$ patients of NASH-HCC and $11.1 \%$ of ASH-HCC were non-cirrhotic at diagnosis of HCC.

3. Many NASH-HCC patients were diagnosed at the symptomatic stage. More rigorous surveillance for HCC in NASH patients than currently practised is needed.
}

\section{INTRODUCTION}

Globally, liver cancer is the 5th and 9th most common malignancy in men and women respectively, representing the 2 nd leading cause of cancer mortality. Significant burden of hepatocellular carcinoma (HCC) is seen in Asia. ${ }^{1,2}$ While viral hepatitis traditionally accounts for most cases of $\mathrm{HCC}$, a burgeoning proportion of HCC is associated with non-alcoholic fatty liver disease (NAFLD) or what was labelled cryptogenic previously. It is increasingly recognised that cryptogenic cirrhosis often represents "burnt-out" NAFLD as a significant number of these patients would have the clinical phenotype comparable with NAFLD, such as higher prevalence of metabolic risk factors, but not the characteristic histopathologic features of NAFLD, which typically disappear with the onset of advanced fibrosis/cirrhosis. ${ }^{4}$ NAFLD is a lifestyle-related condition affecting the liver and is considered as the hepatic man- ifestation of metabolic syndrome due to its association with diabetes mellitus (DM), dyslipidemia, insulin resistance and obesity. NAFLD embodies a spectrum of disease ranging from simple steatosis, considered benign accumulation of lipids within the liver to the more aggressive non-alcoholic steatohepatitis (NASH), characterized by necrosis, inflammation, and fibrosis, with the potential to progress to liver cirrhosis and HCC., ${ }^{5,6}$ NAFLD is regarded as the most common cause of chronic liver disease in the United States and other developed countries. ${ }^{7}$

While the natural history of NAFLD/NASH is still not fully understood, it shares certain similarities with alcoholic liver disease (ALD) and ASH. ${ }^{8}$ In patients with NASH, HCC usually occurs in the setting of liver cirrhosis. However, it is recognized that HCC can develop in non-cirrhotic NASH as well. Over a follow-up period of 7-12 years, the rates of liver-related complications for NAFLD are $3-5 \%$ for cirrhosis, $2 \%$ for jaundice and encephalopathy, $1 \%$ for 
variceal bleeding, and $0.5-1 \%$ for development of $\mathrm{HCC}{ }^{9,10} \mathrm{~A}$ multicenter longitudinal cohort study of biopsy-proven NAFLD reported the outcome of 619 patients over a median follow-up period of 12.6 years - 44 patients (7\%) developed liver-related events including five patients $(0.8 \%)$ diagnosed with HCC. Of the 44 patients who developed the end-stage liver disease, 17 (38.6\%) died from complications of liver disease."

Similarly, ALD is an established risk factor for the development of cirrhosis and HCC. The lifetime risk of development of liver cirrhosis is $8-20 \%$ and that of HCC $3-10 \%$ with ongoing alcohol abuse. ${ }^{12-14}$ Chronic alcohol use of more than $80 \mathrm{~g} /$ day for more than 10 years increases the risk of HCC by 5 -fold. ${ }^{15}$ In a metaanalysis, there was a relative risk of 1.86 of developing HCC with alcohol consumption. The increased risk was present even in patients consuming a low amount of alcohol $\left(25 \mathrm{~g} /\right.$ day) ${ }^{16}$ There is a paucity of data comparing the clinical features of patients with HCC arising from either NAFLD or ALD. In this study, we explored differences in clinical, biochemical and tumor characteristics between NASH-HCC and ASH-HCC. This is important because there is still scant understanding regarding the natural history of NASHHCC. As NASH is closely related to ASH, a comparison between the two etiologies will help us understand specific peculiarities that may help us manage the individual diseases better.

\section{PATIENTS AND METHODS}

\section{Patient selection}

The study cohort comprised patients in a prospective HCC database of the Department of Gastroenterology and Hepatology, Singapore General Hospital. Patients enrolled into the database from 1st July 2000 to 30th May 2013 formed the study cohort. During this period, our HCC registry enrolled 635 patients and after ex-

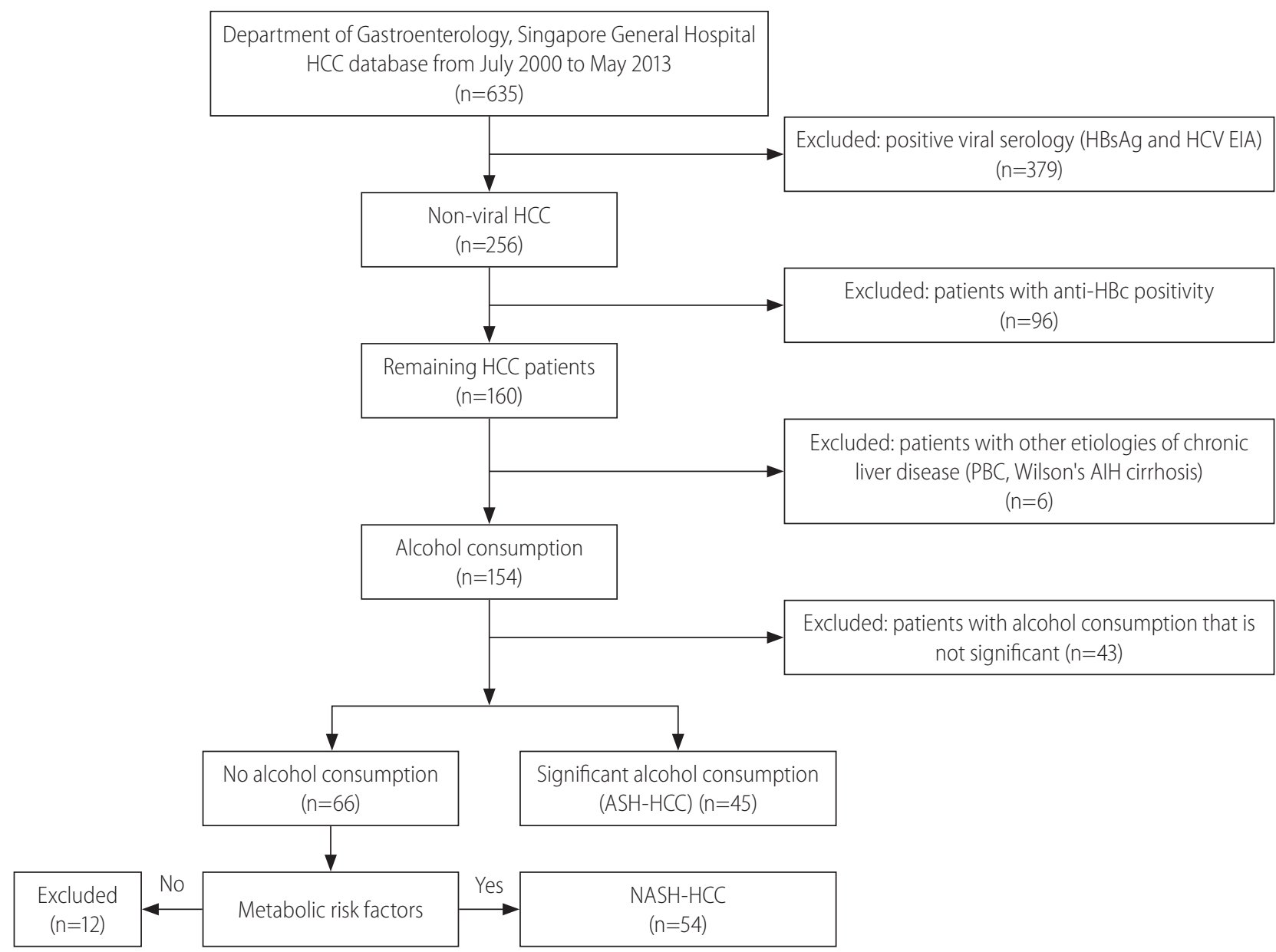

Figure 1. Patient selection flowsheet. HCC, hepatocellular carcinoma; HCV, hepatitis C virus; EIA, enzyme linked immunosorbant assay; PBC, primary biliary cirrosis; AlH, autoimmune hepatitis; $\mathrm{ASH}$, alcoholic steatohepatitis; $\mathrm{NASH}$, non-alcoholic steatohepatitis. 
clusion criteria, 99 patients were included in the study cohort. The patients were followed till 31st October 2015 (see flowchart, Fig. 1).

Diagnosis of HCC was made based on dynamic imaging such as computerized tomography (CT) or magnetic resonance imaging (MRI). ${ }^{17}$ None of the patients included in the study needed a histological assessment for diagnosis of HCC.

Diagnosis of NASH-HCC was based on negative viral serology (negative HBsAg and anti-HCV $\operatorname{lgG}$ ), by the exclusion of other liver diseases like autoimmune hepatitis induced cirrhosis, primary biliary cirrhosis, Wilson's disease and by the absence of significant alcohol intake, in the presence of metabolic syndrome according to International Diabetes Federation and Asia Pacific Working Party on NAFLD. ${ }^{18,19}$ Our definition of NASH-HCC is similar to that of other studies in NASH-HCC. ${ }^{20,21}$

ASH-HCC was diagnosed after negative viral serology and with significant alcohol consumption of more than $60 \mathrm{~g} /$ day in both genders. ${ }^{22}$ To exclude any effect of past hepatitis $B$ infection on the hepatocarcinogenesis in NASH or ASH group, HBclgG data was verified in the study population. All patients with previous hepatits B virus (HBV) exposure as evidenced by $\mathrm{HBclgG}$ positivity were excluded from the study.

There were 54 NASH-HCC and 45 ASH-HCC after consideration of inclusion and exclusion criteria. The diagnosis of cirrhosis was made on the morphological changes seen on dynamic contrast imaging of liver and/or by the presence of radiological, biochemical, endoscopic or clinical features suggestive of cirrhosis or portal hypertension. ${ }^{23-25}$

HCC surveillance was defined as 6-monthly follow up with ultrasound imaging of the liver and serum alpha-fetoprotein (AFP) determination. Not all patients in the study were on HCC surveillance- a number of them presented with symptoms. We stratified the patients as those whose $\mathrm{HCC}$ were diagnosed while they were on a regular surveillance program versus those who were not on a regular surveillance program and whose HCC were diagnosed because they presented with symptoms.

\section{Data collection}

Demographic, anthropometric, clinical and laboratory data were collected at the time of HCC diagnosis and analyzed. Demographic data included age, gender and ethnicity. Anthropometric data included height, weight, waist circumference and body mass index (BMI). Alcohol consumption was quantified based on patients' history and review of medical records. Clinical data reviewed included presence or absence of DM, hypertension, and hyperlipidemia. Hyperlipidemia was considered to be present in a patient with elevated triglycerides $>1.7 \mathrm{mmol} / \mathrm{L}$ and reduced high density lipoprotein- $C_{;}<1.03 \mathrm{mmol} / \mathrm{L}$ in males and $<1.29 \mathrm{mmol} / \mathrm{L}$ in females, or in those patients who were already taking lipid lowering medications. ${ }^{18,19}$ Liver-specific data included liver biochemistry total protein, albumin (Alb), globulin, total bilirubin (Tbil), alkaline phosphatase (ALP), alanine transaminase (ALT), aspartate transaminase (AST), gamma-glutamyltransferase, prothrombin time, international normalized ratio and full blood count including platelet count. If there was cirrhosis, Child-TurcottePugh (CTP) classification and model for end-stage liver disease (MELD) score were recorded.

HCC specific data collected included modality of diagnosis, whether HCC diagnosis was made on surveillance, Barcelona Clinic Liver Cancer (BCLC) staging, and size of the tumor, multifocal or unifocal and treatment types (curative, locoregional or supportive care).

Survival outcome was based on census by our national registry of deaths on 31 October 2015. Liver related mortality was defined by death resulting from liver failure, variceal bleeding, spontaneous bacterial peritonitis or tumor rupture and tumor progression. This study was reviewed and approved by the Singapore General Hospital, Institutional Review Board.

\section{Statistical analysis}

Results are presented as numbers and percentages in separate columns for qualitative data or as the means and standard deviation for quantitative data. Comparisons were by 2 sample $T$-test for quantitative factors and Pearson's chi-square test for qualitative factors. $P$ values less than 0.05 from two-sided tests were considered to be significant. For survival analysis, Kaplan-Meier and Cox regression (Breslow, generalized Wilcoxon) techniques were used. All statistical analyses were performed by using SPSS 24.0 software (SPSS Inc., Chicago, IL, USA).

\section{RESULTS}

\section{Patient characteristics}

The demographic, clinical and biochemical data are summarized in Tables 1 and 2 .

The mean age at diagnosis of HCC of patients in NASH-HCC group was significantly older at $72 \pm 9$ years compared to $66 \pm 9$ 
years in the ASH-HCC group $(P<0.001)$. In the NASH-HCC group there were significantly less male patients compared to the ASHHCC group ( $65 \%$ vs. $96 \%, P<0.001)$. The ethnic composition was significantly different between the two groups with NASH-HCC having significantly more Malay patients compared to the ASHHCC (16.7\% vs. 0\%) and ASH-HCC having significantly more Chinese and Indian patients compared to the NASH-HCC group (Chinese, $91.1 \%$ vs. $79.6 \%$; Indians, $8.9 \%$ vs. $3.7 \% ; P<0.001)$. In the NASH-HCC group, 10 patients (19\%) did not have cirrhosis, whereas in the ASH-HCC group five patients (11\%) did not show features of cirrhosis. This difference was not statistically significant $(P=0.3)$.

The prevalence of DM, hypertension and hyperlipidemia was significantly higher in the NASH-HCC group compared to the ASH-HCC group (see Table 1). The liver biochemistry comprising Alb, Tbil, ALT, AST, and ALP were comparable between the NASH-HCC and ASH-HCC groups.

Table 1. Demographic and clinical comparison between NASH-HCC and ASH-HCC groups

\begin{tabular}{|c|c|c|c|}
\hline \multirow[b]{2}{*}{ Variable } & \multicolumn{2}{|c|}{ Etiology } & \multirow[b]{2}{*}{$P$-value } \\
\hline & $\begin{array}{c}\text { NASH-HCC } \\
(n=54)\end{array}$ & $\begin{array}{c}\text { ASH-HCC } \\
(n=45)\end{array}$ & \\
\hline Gender & & & $<0.001$ \\
\hline Male & $35(64.8)$ & $43(95.6)$ & \\
\hline Female & $19(35.2)$ & $2(4.4)$ & \\
\hline Ethnicity & & & $<0.001$ \\
\hline Chinese & 43 (79.6) & 41 (91.1) & \\
\hline Malay & $9(16.7)$ & $0(0.0)$ & \\
\hline Indian & $2(3.7)$ & $4(8.9)$ & \\
\hline Cirrhosis & & & 0.31 \\
\hline Yes & 44 (81.5) & $40(88.9)$ & \\
\hline No & $10(18.5)$ & $5(11.1)$ & \\
\hline DM & & & $<0.001$ \\
\hline Yes & $42(77.8)$ & $16(35.6)$ & \\
\hline No & $10(18.5)$ & $28(62.2)$ & \\
\hline Hypertension & & & 0.003 \\
\hline Yes & 43 (79.6) & $26(57.8)$ & \\
\hline No & $7(13.0)$ & $18(40.0)$ & \\
\hline Hyperlipidemia & & & $<0.001$ \\
\hline Yes & $29(53.7)$ & $8(17.7)$ & \\
\hline No & $20(37.0)$ & $36(80.0)$ & \\
\hline
\end{tabular}

Values are presented as number (\%).

NASH, non-alcoholic steatohepatitis; HCC, hepatocellular carcinoma; ASH, alcoholic steatohepatitis; DM, diabetes mellitus.
The mean BMI although higher in NASH-HCC group did not differ significantly from the ASH-HCC group. There was no difference in CTP and MELD score between the groups at the diagnosis of $\mathrm{HCC}$.

Both groups showed markedly elevated mean AFP levels with a wide variability. The mean or median AFP values were not significantly different between the two groups.

\section{Details of HCC diagnosis}

Table 3 summarizes the aspects of HCC diagnosis. The diagnosis of HCC was made based on dynamic cross-sectional CT or MRI. A majority of the patient in both groups were diagnosed at the symptomatic stage. Symptomatic stage HCC were patients who presented with symptoms related to the HCC e.g., pain or with decompensated liver disease as opposed to those patients who were asymptomatic and had their HCC diagnosed on surveillance. In the NASH-HCC group, 56\% patients were diagnosed at the symptomatic stage as compared to $53 \%$ patients in ASH-HCC group.

Around $90 \%$ patients of NASH-HCC group were not on surveillance for HCC as compared to $73 \%$ patient of ASH-HCC group. This difference was statistically significant $(P=0.046)$.

Table 2. Biochemical comparison between NASH-HCC and ASH-HCC groups

\begin{tabular}{lccc}
\hline \multirow{2}{*}{ Variable } & \multicolumn{2}{c}{ Etiology } & \\
\cline { 2 - 3 } & $\begin{array}{c}\text { NASH-HCC } \\
(\mathbf{n}=\mathbf{5 4})\end{array}$ & $\begin{array}{c}\text { ASH-HCC } \\
(\mathbf{n}=\mathbf{4 5})\end{array}$ & P-value \\
\hline Age & $72 \pm 9$ & $66 \pm 9$ & $<0.001$ \\
\hline BMI $\left(\mathrm{kg} / \mathrm{m}^{2}\right)$ & $27.2 \pm 4.6$ & $23.7 \pm 5.0$ & 0.08 \\
\hline Albumin $(\mathrm{g} / \mathrm{L})$ & $29 \pm 8$ & $30 \pm 5$ & 0.29 \\
\hline Bilirubin $($ umol/L) & $44 \pm 59$ & $47 \pm 63$ & 0.11 \\
\hline ALP $($ IU/L) & $143 \pm 91$ & $169 \pm 124$ & 0.45 \\
\hline ALT $($ IU/L) & $40 \pm 21$ & $41 \pm 23$ & 0.88 \\
\hline AST $($ IU/L) & $91 \pm 109$ & $73 \pm 50$ & 0.96 \\
\hline GGT (IU/L) & $73 \pm 135$ & $179 \pm 273$ & 0.06 \\
\hline AFP (ug/L) & $8,241 \pm 19,343$ & $21,633 \pm 107,187$ & 0.41 \\
CTP score & $7.67 \pm 1.95$ & $7.73 \pm 1.97$ & 0.98 \\
\hline MELD & $12.55 \pm 5.1$ & $13.29 \pm 4.9$ & 0.48 \\
\hline
\end{tabular}

Values are presented as mean \pm standard deviation.

NASH, non-alcoholic steatohepatitis; HCC, hepatocellular carcinoma; ASH, alcoholic steatohepatitis; BMI, body mass index; ALP, alkaline phosphatase; ALT, alanine transaminase; AST, aspartate transaminase; GGT, gamma glutamyl transferases; AFP, alpha-fetoprotein; CTP, Child-Turcotte-Pugh; MELD, model for end-stage liver disease. 


\section{Tumor characteristics}

Tumour Characteristics are summarised in Table 4. There were no significant differences between the two groups in the prevalence of multifocal HCC and of portal vein invasion (see Table 4). Lymph node invasion was seen more frequently in ASH-HCC compared to NASH-HCC (22\% vs. $7.5 \%, P=0.031)$. BCLC and Tumor lymph Node Metastasis (TNM) staging were similar between the two groups. Due to the retrospective nature of study, some data regarding the TNM classification and $\mathrm{BCLC}$ staging was missing from our dataset.

\section{HCC treatment}

HCC treatment modalities are summarized in Table 4. Only 20\% patients in ASH-HCC group received treatment with curative intent (liver transplantation, liver resection or radiofrequency ablation) compared to $26 \%$ in NASH-HCC group $(P=0.41)$. More than $50 \%$ of patients in both groups were not suitable for any treatment and were accorded best supportive care.

\section{HCC survival outcomes}

The survival outcome data for 96 patients (51 NASH-HCC and $45 \mathrm{ASH}-\mathrm{HCC}$ ) were available. Three patients in the NASH - HCC group were foreigners who returned to their home countries after HCC diagnosis and initial treatment. Six patients (11.8\%) in NASH-HCC group and three patients $(6.7 \%)$ in the ASH-HCC

Table 3. Differences in aspects of HCC diagnosis between NASH-HCC and ASH-HCC groups

\begin{tabular}{lccc}
\hline \multirow{2}{*}{ Variable } & \multicolumn{2}{c}{ Etiology } & \\
\cline { 2 - 3 } & $\begin{array}{c}\text { NASH-HCC } \\
(\mathbf{n}=54)\end{array}$ & $\begin{array}{c}\text { ASH-HCC } \\
(\mathbf{n}=\mathbf{4 5})\end{array}$ & \\
\hline $\begin{array}{c}\text { On screening/ } \\
\text { surveillance }\end{array}$ & & & 0.046 \\
Yes & $6(11.1)$ & $12(26.7)$ & \\
No & $48(88.9)$ & $33(73.3)$ & \\
Symptomatic at & & & 0.82 \\
diagnosis & & & \\
Yes & $30(55.6)$ & $24(53.3)$ & \\
No & $24(44.4)$ & $21(46.7)$ & \\
\hline
\end{tabular}

Values are presented as number (\%).

HCC, hepatocellular carcinoma; NASH, non-alcoholic steatohepatitis; ASH, alcoholic steatohepatitis.
Table 4. Differences in the tumor characteristics between NASH-HCC and ASH-HCC groups

\begin{tabular}{|c|c|c|c|}
\hline \multirow[b]{2}{*}{ Variable } & \multicolumn{2}{|c|}{ Etiology } & \multirow[b]{2}{*}{$P$-value } \\
\hline & $\begin{array}{l}\text { NASH-HCC } \\
(n=54)\end{array}$ & $\begin{array}{c}\text { ASH-HCC } \\
(n=45)\end{array}$ & \\
\hline Number of lesions & & & 0.99 \\
\hline Single & $31(57.4)$ & $26(57.8)$ & \\
\hline Multiple & $20(37.0)$ & $17(37.8)$ & \\
\hline Diffuse & $2(3.7)$ & $2(4.4)$ & \\
\hline Unknown & $1(1.8)$ & $0(0.0)$ & \\
\hline Portal vein involvement & & & 0.81 \\
\hline No & $35(64.8)$ & $28(62.2)$ & \\
\hline Yes & $18(33.3)$ & $16(35.6)$ & \\
\hline Unknown & $1(1.9)$ & $1(2.2)$ & \\
\hline Lymph node involvement & & & 0.03 \\
\hline No & $50(92.6)$ & $34(75.6)$ & \\
\hline Yes & $4(7.4)$ & $10(22.2)$ & \\
\hline Unknown & $0(0.0)$ & $1(2.2)$ & \\
\hline BCLC stage & & & 0.26 \\
\hline 0 & $1(6.2)$ & $0(0.0)$ & \\
\hline A & $5(31.3)$ & $3(42.9)$ & \\
\hline B & $2(12.5)$ & $1(14.2)$ & \\
\hline C & $2(12.5)$ & $3(42.9)$ & \\
\hline D & $6(37.5)$ & $0(0.0)$ & \\
\hline TNM staging (AJCC 7ed) & & & 0.37 \\
\hline । & $9(56.3)$ & $2(28.6)$ & \\
\hline$\|$ & $2(12.5)$ & $1(14.3)$ & \\
\hline$\| \mathrm{A}$ & $2(12.5)$ & $2(28.6)$ & \\
\hline$\| \mathrm{II}$ & $2(12.5)$ & $0(0.0)$ & \\
\hline$\| I C$ & $0(0.0)$ & $0(0.0)$ & \\
\hline IVA & $0(0.0)$ & $0(0.0)$ & \\
\hline IVB & $1(6.3)$ & $2(28.6)$ & \\
\hline Treatment type & & & 0.41 \\
\hline Curative & $14(25.9)$ & $9(20.0)$ & \\
\hline Loco-regional therapy & $11(20.3)$ & $10(22.2)$ & \\
\hline Systemic chemotherapy & $1(1.9)$ & $2(4.4)$ & \\
\hline Best supportive care & $28(51.8)$ & $24(53.3)$ & \\
\hline
\end{tabular}

Values are presented as number (\%). There were missing data in the registry regarding the BCLC and TNM staging. Staging data was available for 23 patients, which is presented in the table.

HCC, hepatocellular carcinoma; NASH, non-alcoholic steatohepatitis; ASH, alcoholic steatohepatitis; BCLC, Barcelona Clinic Liver Cancer; TNM, Tumor Iymph Node Metastasis; AJCC, American Joint Committee on Cancer. 
group were alive at the time of census. Survival Comparison between the groups is shown in Table 5 .

Liver related mortality was defined by death resulting from liver failure, variceal bleeding, spontaneous bacterial peritonitis, tumor rupture or tumor progression. Other causes of mortality in NASHHCC group included septicemia in five (10\%), metastatic cancer of other organs in two (4\%), and DM induced come in one patients (2\%). In the ASH-HCC group septicemia was cause of mortality in two (4.4\%) and metastatic cancer of other organs in one patients (2.2\%). Table 6 shows the other (excluding liver related) causes of mortality in both groups.

\section{Overall survival (OS)/all-cause mortality}

The median OS of NASH-HCC group was $13 \pm 2.5$ months and that of ASH-HCC was $7 \pm 1.6$ months ( $P=0.12$ ) (Fig. 2). On multivariate logistic regressions, the significant determinant of allcause mortality were CTP score $(P<0.001)$ and tumor stages III $(P=0.014)$ and IV $(P<0.001)$. Age was not a significant factor of $O S$ in the multivariable analysis $(P=0.4)$, neither was presence or

Table 5. Survival comparison between the groups

\begin{tabular}{lccc}
\hline & N & All-cause mortality & Liver related mortality \\
\hline NASH-HCC & 51 & $45(88)$ & $36(70)$ \\
ASH-HCC & 45 & $42(93)$ & $38(84)$ \\
Total & 96 & $87(90)$ & $74(77)$ \\
\hline
\end{tabular}

Values are presented as number (\%).

$\mathrm{N}$, total number of patients; NASH, non-alcoholic steatohepatitis; HCC, hepatocellular carcinoma; ASH, alcoholic steatohepatitis.

Table 6. Causes of non-liver-related mortality

\begin{tabular}{ll}
\hline & Value \\
\hline NASH-HCC (9/51) & \\
Septicemia & $5(10)$ \\
Other metastatic cancers (colon, 1; unknown primary, 1) & $2(4.4)$ \\
DM coma & $1(2)$ \\
Multi-organ failure of unknown cause & $1(1)$ \\
ASH-HCC (4/45) & \\
Septicemia & $2(4.4)$ \\
Other metastatic cancers (prostate, 1) & $1(2.2)$ \\
Multi-organ failure of unknown cause & $1(2.2)$ \\
\hline
\end{tabular}

Values are presented as number (\%).

NASH, non-alcoholic steatohepatitis; HCC, hepatocellular carcinoma; DM, diabetes mellitus; ASH, alcoholic steatohepatitis. absence of DM, cirrhosis, portal vein or lymph node involvement (Tables 7 and 8).

\section{Liver related mortality}

With regards to liver related mortality, the median survival of NASH-HCC group was significantly better than ASH-HCC $(19 \pm 10.3$ vs. $8 \pm 1.3$ months, $P=0.047$ ) (Fig. 3). Multivariable logistic regression showed CTP score $(\mathrm{p}=0.003)$ and tumor stages III $(P=0.025)$ and IV ( $P=0.001)$ as the significant determinant of liver related mortality (Tables 7 and 8). Patient age at diagnosis, presence or absence of DM, cirrhosis, portal vein involvement or lymph node involvement were not significant determinants of liver-related mortality.

\section{DISCUSSION}

The main findings of this study are that the NASH-HCC patients were older at diagnosis, had significantly more metabolic risk factors and were diagnosed less frequently on surveillance or screening as compared to ASH-HCC.

There is often concern over the diagnosis of NASH-related HCC. NASH and metabolic syndrome are closely intertwined, where insulin resistance plays a key role in both disease entities and NASH assumed to be the hepatic manifestation of metabolic syndrome. In an earlier study of 65 patients with NASH, Chitturi et al. ${ }^{26}$

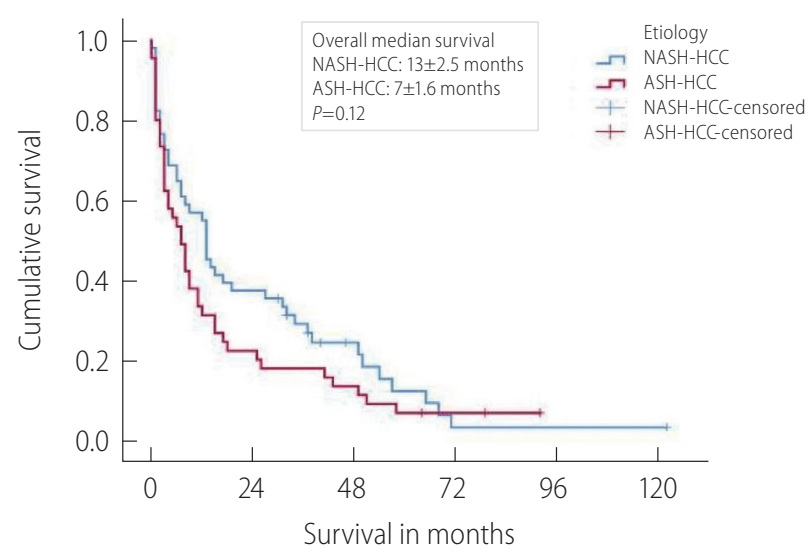

Number of patients at risk

$\begin{array}{lrrrrrr}\text { NASH-HCC } & 51 & 19 & 13 & 6 & 6 & 6 \\ \text { ASH-HCC } & 45 & 9 & 5 & 3 & 3 & 3\end{array}$

Figure 2. Kaplan Meier overall survival analysis between the NASH-HCC and ASH-HCC groups. NASH, non-alcoholic steatohepatitis; HCC, hepatocellular carcinoma; ASH, alcoholic steatohepatitis. 
Table 7. Univariate Cox regression

\begin{tabular}{|c|c|c|c|c|}
\hline \multirow{2}{*}{ Variable } & \multicolumn{2}{|c|}{ All-cause mortality } & \multicolumn{2}{|c|}{ Liver-related mortality } \\
\hline & HR $(95 \% \mathrm{Cl})$ & $P$-value & $\mathrm{HR}(95 \% \mathrm{Cl})$ & $P$-value \\
\hline \multicolumn{5}{|l|}{ Etiology } \\
\hline NASH-HCC & 1.00 & & 1.00 & \\
\hline ASH-HCC & $1.31(0.94-1.68)$ & 0.09 & $1.48(1.31-2.14)$ & 0.03 \\
\hline Age & $1.01(0.99-1.04)$ & 0.39 & $1.01(0.98-1.03)$ & 0.58 \\
\hline \multicolumn{5}{|l|}{ Gender } \\
\hline Female & 1.00 & & 1.00 & \\
\hline Male & $1.40(0.83-2.36)$ & 0.21 & $1.44(0.81-2.54)$ & 0.21 \\
\hline \multicolumn{5}{|l|}{$\mathrm{DM}$} \\
\hline No & 1.00 & & 1.00 & \\
\hline Yes & $1.14(0.74-1.74)$ & 0.56 & $1.07(0.67-1.70)$ & 0.77 \\
\hline \multicolumn{5}{|l|}{ HTN } \\
\hline No & 1.00 & & 1.00 & \\
\hline Yes & $1.21(0.76-1.91)$ & 0.43 & $1.08(0.66-1.76)$ & 0.76 \\
\hline \multicolumn{5}{|l|}{ HLD } \\
\hline No & 1.00 & & 1.00 & \\
\hline Yes & $0.86(0.56-1.33)$ & 0.49 & $0.77(0.48-1.24)$ & 0.28 \\
\hline \multicolumn{5}{|l|}{ Cirrhosis } \\
\hline No & 1.00 & & 1.00 & \\
\hline Yes & $0.77(0.49-1.22)$ & 0.27 & $0.67(0.39-1.38)$ & 0.37 \\
\hline Albumin & $0.93(0.90-0.96)$ & $<0.001$ & $0.94(0.91-0.98)$ & 0.001 \\
\hline ALP & $1.005(1.003-1.007)$ & $<0.001$ & 1.005 (1.003-1.007) & $<0.001$ \\
\hline ALT & $1.001(0.99-1.01)$ & 0.90 & $1.004(0.99-1.02)$ & 0.50 \\
\hline AST & $1.007(1.004-1.009)$ & $<0.001$ & $1.007(1.005-1.009)$ & $<0.001$ \\
\hline GGT & $1.001(1.00009-1.002)$ & 0.04 & $1.001(1.0002-1.003)$ & 0.02 \\
\hline AFP & $1.000003(1.000001-1.000006)$ & 0.01 & $1.000004(1.000001-1.000006)$ & 0.004 \\
\hline Total bilirubin & $1.005(1.002-1.009)$ & 0.004 & $1.006(1.002-1.009)$ & 0.001 \\
\hline Serum creatinine & $1.003(1.0001-1.005)$ & 0.04 & $1.002(0.999-1.005)$ & 0.23 \\
\hline MELD score & $1.05(1.004-1.09)$ & 0.03 & $1.05(1.007-1.10)$ & 0.02 \\
\hline CTP score & $1.29(1.16-1.44)$ & $<0.001$ & $1.26(1.12-1.42)$ & $<0.001$ \\
\hline \multicolumn{5}{|l|}{ Portal vein involved } \\
\hline No & 1.00 & & 1.00 & \\
\hline Yes & $3.35(2.08-5.40)$ & $<0.001$ & $3.79(2.27-6.35)$ & $<0.001$ \\
\hline \multicolumn{5}{|c|}{ Lymph node involved } \\
\hline No & 1.00 & & 1.00 & \\
\hline Yes & $4.35(2.29-8.25)$ & $<0.001$ & $3.86(1.90-7.84)$ & $<0.001$ \\
\hline \multicolumn{5}{|c|}{ TNM staging (AJCC 7th ed) } \\
\hline । & 1.00 & & 1.00 & \\
\hline$\|$ & $1.88(1.004-3.52)$ & 0.05 & $1.82(0.91-3.66)$ & 0.09 \\
\hline III & $3.00(1.75-5.14)$ & $<0.001$ & $2.94(1.64-5.28)$ & $<0.001$ \\
\hline IV & $8.58(4.08-18.03)$ & $<0.001$ & $9.50(4.30-20.97)$ & $<0.001$ \\
\hline
\end{tabular}

HR, hazard ratio; $\mathrm{Cl}$, confidence interval; NASH, non-alcoholic steatohepatitis; HCC, hepatocellular carcinoma; ASH, alcoholic steatohepatitis; DM, diabetes mellitus; HTN, hypertension; HLD, hyperlipidaemia; ALP, alkaline phosphatase; ALT, alanine transaminase; AST, aspartate transaminase; GGT, gamma glutamyl transferases; AFP, alpha-fetoprotein; MELD, model for end-stage liver disease; CTP, Child-Turcotte-Pugh; TNM, Tumor lymph Node Metastasis; AJCC, American Joint Committee on Cancer. 
Table 8. Multivariable Cox regression

\begin{tabular}{|c|c|c|c|c|}
\hline \multirow{2}{*}{ Variable } & \multicolumn{2}{|c|}{ All-cause mortality } & \multicolumn{2}{|c|}{ Liver-related mortality } \\
\hline & $\mathrm{HR}(95 \% \mathrm{Cl})$ & $P$-value & $\mathrm{HR}(95 \% \mathrm{Cl})$ & $P$-value \\
\hline \multicolumn{5}{|l|}{ Etiology } \\
\hline NASH-HCC & 1.00 & & 1.00 & \\
\hline ASH-HCC & $1.28(0.73-1.87)$ & 0.08 & $1.56(1.21-1.95)$ & 0.04 \\
\hline Age & $1.03(0.9995-1.06)$ & 0.40 & $1.03(0.99-1.06)$ & 0.32 \\
\hline \multicolumn{5}{|l|}{ DM } \\
\hline No & 1.00 & & 1.00 & \\
\hline Yes & $0.91(0.55-1.50)$ & 0.70 & $0.90(0.52-1.55)$ & 0.70 \\
\hline \multicolumn{5}{|l|}{ Cirrhosis } \\
\hline No & 1.00 & & 1.00 & \\
\hline Yes & $0.74(0.43-1.28)$ & 0.28 & $0.67(0.37-1.23)$ & 0.20 \\
\hline CTP & $1.28(1.12-1.46)$ & $<0.001$ & $1.25(1.08-1.45)$ & 0.003 \\
\hline \multicolumn{5}{|c|}{ Portal vein involved } \\
\hline No & 1.00 & & 1.00 & \\
\hline Yes & $1.47(0.78-2.76)$ & 0.23 & $1.78(0.89-3.55)$ & 0.10 \\
\hline \multicolumn{5}{|c|}{ Lymph node involved } \\
\hline No & 1.00 & & 1.00 & \\
\hline Yes & $1.07(0.47-2.41)$ & 0.88 & $0.78(0.32-1.94)$ & 0.60 \\
\hline \multicolumn{5}{|c|}{ TNM staging (AJCC 7th ed) } \\
\hline । & 1.00 & & 1.00 & \\
\hline$\|$ & $2.00(0.96-4.16)$ & 0.06 & $1.73(0.76-3.93)$ & 0.19 \\
\hline III & $2.31(1.19-4.51)$ & 0.01 & $2.30(1.11-4.76)$ & 0.03 \\
\hline IV & $6.38(2.19-18.57)$ & 0.001 & $7.13(2.28-22.29)$ & 0.001 \\
\hline
\end{tabular}

HR, hazard ratio; $\mathrm{Cl}$, confidence interval; NASH, non-alcoholic steatohepatitis; HCC, hepatocellular carcinoma; ASH, alcoholic steatohepatitis; DM, diabetes mellitus; CTP, Child-Turcotte-Pugh; TNM, Tumor lymph Node Metastasis; AJCC, American Joint Committee on Cancer.

showed that virtually all subjects (98\%) were insulin resistant. It is commonly accepted that in the context of liver disease, presence of metabolic syndrome connotes presence of NASH, in the absence of other etiologies of chronic liver disease, such as alcohol or viral related causes. Separately, Bugianesi et al. ${ }^{27}$ demonstrated that features suggestive of the metabolic syndrome, such as DM, dyslipidemia, obesity and insulin resistance were reported more frequently in patients with HCC arising in the setting of cryptogenic/NASH cirrhosis than in matched controls.

We used a definition of NASH-HCC based on the above considerations and which is similar that used in other studies on NASH HCC. ${ }^{20,21}$

The biochemical parameters were not significantly different between the groups. Interestingly a number of patients in NASHHCC (18.5\%) and ASH-HCC (11.1\%) were not cirrhotic at diagnosis of HCC. Since many NASH-HCC patients were diagnosed at symp- tomatic stage and not by surveillance or screening a more rigorous screening and surveillance methods is required for NASH-HCC patients than currently practiced. In terms of overall mortality both the groups were the same but NASH-HCC patients fared better than ASH-HCC in terms of liver related mortality. Our study also showed that significantly less NASH-HCC patients die from liver-related mortality compared to ASH-HCC. Instead, more NASH-HCC patients also died from septicemia unrelated to liver cancer, diabetes induced coma and metastatic disease of other organs as compared to ASH-HCC group.

Even though histologically NASH and ASH are quite similar they are different in the natural history and liver-related complication rates. ${ }^{28,29}$ ASH and NASH can be considered as diseases with differing aetiologies but of a similar morphologic spectrum. One of the main complications of both NASH and ASH is the development of HCC. 


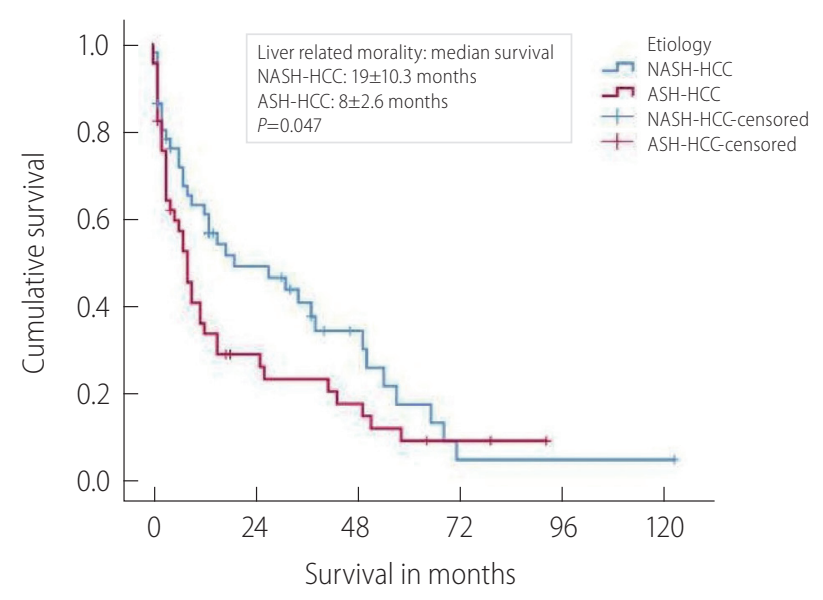

Number of patients at risk

$\begin{array}{lllllll}\text { NASH-HCC } & 51 & 27 & 22 & 15 & 15 & 15\end{array}$

$\begin{array}{lllllll}\text { ASH-HCC } & 45 & 13 & 9 & 7 & 7 & 7\end{array}$

Figure 3. Kaplan Meier survival curve: liver-related mortality. NASH, non-alcoholic steatohepatitis; HCC, hepatocellular carcinoma; ASH, alcoholic steatohepatitis.

The liver undergoes simultaneous regeneration and fibrosis in response to injury. These divergent responses lead to distinctive pathways of hepatocarcinogenesis. Potential mechanisms of fibrosis-dependent carcinogenesis include increased integrin signalling by the fibrotic matrix, paracrine signalling between hepatic stellate cells and hepatocytes, increased stromal stiffness, growth factor sequestration by extracellular matrix, and reduced tumor surveillance by natural killer and natural killer T cells. ${ }^{30}$ Apart from the fibrosis induced hepatocarcinogenesis, chronic alcohol consumption interferes with several host anti-tumor mechanisms, thereby facilitating hepatocyte proliferation and tumorigenesis. The major mechanisms of alcohol-induced HCC include pathways of ethanol metabolism, alcohol-induced oxidative stress and hypomethylation of DNA, and interplay of alcohol with iron elevation, retinoid metabolism, the immune system, inflammatory pathways, and neoangiogenesis. ${ }^{31,32}$ The understanding of hepatocarcinogenesis in NASH although still evolving, is believed to originate from deregulated one-carbon metabolism, nuclear factor $\mathrm{kB}$ proteins, phosphatase and tensin homolog (PTEN), microRNA instability and telomere shortening. ${ }^{33}$

Consistent with published literature our patients with NASHHCC were significantly older than patients with ASH-HCC with most of the patients in NASH-HCC group in their 7th decade of life whereas patients in ASH-HCC group were a decade younger. ${ }^{34-36}$ Older age at diagnosis of NASH-HCC cohort has clinical implications. HCC screening and surveillance strategies should in- clude elderly patients and the treatment strategies should also address treatment approaches in elderly patients. NASH-HCC group comprised significantly more female patients compared to the ASH-HCC group, which is consistent with the alcohol consumption pattern worldwide. ${ }^{37-39}$

Interestingly our study showed that $18 \%$ of the patients in NASH-HCC group and $11 \%$ of the patients in ASH-HCC group had no apparent signs of cirrhosis on radiological examination. This observation echoes previously published literature demonstrating the occurrence of NASH related HCC in the absence of cirrhosis. There is an increasing body of evidence showing that HCC can occur in patients with non-cirrhotic NASH. Two recent large studies from Japan of $292^{40}$ and 87 patients ${ }^{41}$ with NAFLD and HCC reported that non-cirrhotic patients comprised $38 \%$ and $49 \%$ respectively of the cases of HCC. A recent meta-analysis of 168,571 subjects revealed that non-cirrhotic NASH subjects were 2.61 times significantly more likely to develop HCC compared to noncirrhotic subjects of other etiologies. ${ }^{42}$ Another study from the USA reported $34.6 \%$ of patients in NAFLD-HCC group and $11.1 \%$ of ALD-related HCC as not having cirrhosis at diagnosis. ${ }^{43}$ This is consistent with the results of our study. One possible reason for HCC occurring in non-cirrhotic ASH is that the main underlying mechanism for hepatocarcinogenesis in ALD is the oxidative stress of chronic inflammation coupled with induction of cytochrome P-450 by alcohol leading to reactive oxygen species production that in turn causes lipid peroxidation, DNA damage, iron overload and immune system dysfunction. ${ }^{44}$ This hepatocarcinogenesis pathway is independent of fibrosis and cirrhosis. Nevertheless, the development of HCC in ASH/ALD without cirrhosis may not be widely recognised and warrants further studies. ${ }^{45}$

Another notable observation in our study was that majority of patients in both groups were symptomatic at diagnosis. Only $11.1 \%$ of the patients in NASH-HCC group were diagnosed on surveillance imaging compared to $26.7 \%$ of the patients in ASHHCC group. This finding underlines the fact that NASH patients may not be receiving adequate surveillance for liver-related complications since there is no consensus regarding HCC surveillance in NASH. Major society guidelines recommend 6 monthly ultrasonography surveillance for HCC on at-risk population, i.e., in cirrhotic patients and HBV positive patients in endemic areas. ${ }^{17,46}$ Considering the fact that approximately $20 \%$ of patients with NASH develop HCC in a non-cirrhosis setting, ${ }^{47}$ which is also corroborated by the data of our study, where $18 \%$ of patients in NASH-HCC group were non-cirrhotic, is a need to identify these NASH patients who are at increased risk of developing HCC so 
that appropriate surveillance strategies can be formulated. Currently, this subset of the patient is poorly defined.

To reduce the morbidity and mortality associated with late detection of HCC, we propose that patients with ALD as well as those with NAFLD/NASH should have fibrosis assessment and those with advanced/bridging fibrosis ( $>$ F3) or cirrhosis should be on 6-monthly surveillance for HCC. This strategy is echoed in the recently published HCC guidelines from the European Association of study of liver diseases. ${ }^{17}$ Individualized intensive screening programs based on risk prediction of HCC like ADRESS-HCC ${ }^{48}$ needs further validation in different cohorts.

Approximately $40 \%$ of the patients in both groups had multifocal or diffuse HCC. Portal vein involvement and tumor extent were similar between the NASH-HCC and ASH-HCC groups. Majority of the patients in both groups were diagnosed late in the course of disease and more than $50 \%$ of them were symptomatic at presentation. As such, treatment options were limited in many patients. Only 4.4\% patients in the ASH-HCC group and 13\% patients in the NASH-HCC group underwent curative resection. None of the patients in either group could receive liver transplantation due to advanced disease and non-availability of a liver graft. Similar experiences in the treatment of NASH-HCC were reported by Weinmann and colleagues, ${ }^{49}$ who in a cohort of $45 \mathrm{bi}$ opsy proven NASH-HCC patients found only $17.8 \%$ went for resection, $4.4 \%$ for liver transplant after bridging therapy and $73.3 \%$ for locoregional therapy or supportive care. Recently data from a large French cohort consisting of 582 alcohol induced HCC reported that treatment with curative intent was less likely to happen in alcohol induced HCC, only $16.3 \%$ patient could undergo treatment with curative intent. ${ }^{50}$

Our study showed that for all-cause mortality, although the median OS of NASH-HCC was numerically better than that of ASHHCC (13 months vs. 7 months), it was not statistically significant $(P=0.12)$. However, the median survival of NASH-HCC group was significantly better than ASH-HCC (19 months vs. 8 months) for liver related mortality $(P=0.047)$. Patient's age at diagnosis of HCC did not affect the median OS or liver-related mortality of both groups. This etiological difference of survival between the groups is consistent with previously published NASH-HCC survival data from Weinmann and colleagues ${ }^{49}$ and alcohol induced HCC survival data by Costentin and colleagues ${ }^{50}$ who reported the lead time adjusted median OS of 5.7 months in 528 alcohol induced HCC patients.

The reason for difference in liver related mortality between the groups, with the NASH-HCC group having significantly better sur- vival, is that the alcohol related HCC patients were likely to be imbibing alcohol till the point of admission, with a fair number of them having florid alcoholic hepatitis which played an important part in their liver related mortality. However, the OS which was not statistically different between the two groups (although showing a better trend for NASH-HCC group) was due to the fact that after the initial period, it's the tumour stage and grade that were predominant factors in determining the mortality.

Our study is limited by its retrospective nature and single center data. Alcohol quantification was via patient self-reporting and electronic medical records, which cannot exclude a certain degree of misclassification. Nevertheless, this study provides important information on HCC attributed to two well established etiologies of chronic liver disease in an Asian centre. It is the first study to compare the difference in demographics, clinical, biochemical, tumor characteristics and survival outcomes between NASH and ASH related HCC. Data collection through interconnected electronic record system and mandatory national mortality reporting makes our data robust. Further prospective studies should look for ways to improve OS in both groups.

NASH-HCC and ASH-HCC differ in their etiology and pathogenesis. Despite these differences, liver and tumor characteristics were comparable between NASH-HCC and ASH-HCC. Patients with NASH-HCC had more metabolic risk factors and were older at diagnosis compared to ASH-HCC. Most patients in both groups were diagnosed late and were not amenable to curative or locoregional therapies. OS of NASH-HCC and ASH-HCC were not statistically different. Better characterization of the at-risk population of patients with NASH and ASH are needed to optimize screening, surveillance, and management of NASH and ASH related HCC.

\section{Authors' contribution}

RK, BBGG and CKT conceptualised the study, collected and analysed the data and wrote the manuscript, JWK performed detailed statistical analyses of the data, PEC collected and analysed the data, reviewed and refined the manuscript.

\section{Acknowledgements}

The authors wish to acknowledge all the doctors in the Department of Gastroenterology and Hepatology, Singapore General Hospital, for allowing their patients to be enrolled into the HCC registry. 


\section{Conflicts of Interest}

The authors have no conflicts to disclose.

\section{REFERENCES}

1. El-Serag HB, Rudolph KL. Hepatocellular carcinoma: epidemiology and molecular carcinogenesis. Gastroenterology 2007;132:25372576.

2. Goh GB, Chang PE, Tan CK. Changing epidemiology of Hepatocellular carcinoma in Asia. Best Pract Res Clin Gastroenterol 2015;29:919-928.

3. Bosch FX, Ribes J, Cléries R, Díaz M. Epidemiology of hepatocellular carcinoma. Clin Liver Dis 2005;9:191-211, v.

4. Cadwell SH, Lee VD, Kleiner DE, Al-Osaimi AM, Argo CK, Northup $P G$, et al. NASH and cryptogenic cirrhosis: a histological analysis. Ann Hepatol 2009;8:346-352.

5. Chalasani N, Younossi Z, Lavine JE, Charlton M, Cusi K, Rinella M, et al. The diagnosis and management of non-alcoholic fatty liver disease: practice guidance from the American Association for the Study of Liver Diseases. Hepatology 2018;67:328-357.

6. Matteoni CA, Younossi ZM, Gramlich T, Boparai N, Liu YC, McCollough AJ. Nonalcoholic fatty liver disease: a spectrum of clinical and pathological severity. Gastroenterology 1999;116:1413-1419.

7. Albhaisi S, Sanyal A. Recent advances in understanding and managing non-alcoholic fatty liver disease. F1000Res 2018;7(F1000 Faculty Rev):720.

8. Tannapfel A, Denk H, Dienes HP, Langner C, Schiemacher P, Trauner $M$, et al. Histopathological diagnosis of non-alcoholic and alcoholic fatty liver disease. Virchows Arch 2011;458:511-523.

9. Adams LA, Lymp JF, St Sauver J, Sanderson SO, Lindor KD, Angulo P, et al. The natural history of nonalcoholic fatty liver disease: a population-based cohort study. Gastroenterology 2005;129:113-121.

10. Ekstedt M, Franzén LE, Mathiesen UL, Thorelius L, Holmqvist M, Bodemar $G$, et al. Long-term follow-up of patients with NAFLD and elevated liver enzymes. Hepatology 2006;44:865-873.

11. Angulo P, Kleiner DE, Dam-Larsen S, Adams LA, Bjornsson ES, Charatcharoenwitthaya $P$, et al. Liver fibrosis, but no other histologic features, is associated with long-term outcomes of patients with the nonalcoholic fatty liver disease. Gastroenterology 2015;149:389397.e10.

12. Askgaard G, Grønbaek M, Kjær MS, Thønneland A, Toistrup JS. Alcohol drinking pattern and risk of alcoholic liver cirrhosis: a prospective cohort study. J Hepatol 2015;62:1061-1067.

13. Åberg F, Helenius-Hietala J, Puukka P, Jula A. Binge drinking and the risk of liver events: a population-based cohort study. Liver Int 2017;37:1373-1381.
14. Singal AK, Bataller R, Ahn J, Kamath PS, Shah VH. ACG clinical guideline: alcoholic liver disease. Am J Gastroenterol 2018;113:175194.

15. Donato F, Tagger A, Gelatti U, Parrinello G, Boffetta P, Albertini A, et al. Alcohol and hepatocellular carcinoma: the effect of lifetime intake and hepatitis virus infections in men and women. Am J Epidemiol 2002;155:323-331.

16. Bagnardi V, Blangiardo M, La Vecchia C, Corrao G. Alcohol consumption and the risk of cancer: a meta-analysis. Alcohol Res Health 2001;25:263-270.

17. European Association for the Study of the Liver. EASL clinical practice guidelines: management of hepatocellular carcinoma. J Hepatol 2018;69:182- 236.

18. Alberti KGMM, Zimmet P, Shaw J. Metabolic syndrome-a new world-wide definition. A Consensus Statement from the International Diabetes Federation. Diabet Med 2006;23:469-480.

19. Wong VW, Chan WK, Chitturi S, Chawla Y, Dan YY, Duseja A, Fan J, et al. Asia-Pacific Working Party on non-alcoholic fatty liver disease guidelines 2017-Part 1: definition, risk factors and assessment. J Gastroenterol Hepatol 2017;33:70-85.

20. Younossi ZM, Otgonsuren M, Henry L, Venkatesan C, Mishra A, Erario $\mathrm{M}$, et al. Association of nonalcoholic fatty liver disease (NAFLD) with hepatocellular carcinoma (HCC) in the United States from 2004 to 2009. Hepatology 2015;62:1723-1730.

21. Lee SS, Jeong SH, Byoun YS, Chung SM, Seong MH, Sohn HR, et al. Clinical features and outcome of cryptogenic hepatocellular carcinoma compared to those of viral and alcoholic hepatocellular carcinoma. BMC Cancer 2013;13:335.

22. European Association for the Study of the Liver. EASL clinical practice guidelines: management of alcohol-related liver disease. J Hepatol 2018;69:154-181.

23. Colli A, Fraquelli M, Andreoletti M, Marino B, Zuccoli E, Conte D. Severe liver fibrosis or cirrhosis: accuracy of US for detection--analysis of 300 cases. Radiology 2003;227:89-94.

24. Kudo M, Zheng RQ, Kim SR, Okabe Y, Osaki Y, lijima H, et al. Diagnostic accuracy of imaging for liver cirrhosis compared to histologically proven liver cirrhosis. A multicenter collaborative study. Intervirology 2008;51 Suppl 1:17-26.

25. Yeom SK, Lee CH, Cha SH, Park CM. Prediction of liver cirrhosis, using diagnostic imaging tools. World J Hepatol 2015;7:2069-2079.

26. Chitturi S, Abeygunasekera S, Farrell GC, Holmes-Walker J, Hui JM, Fung $\mathrm{C}$, et al. NASH and insulin resistance: insulin hypersecretion and specific association with the insulin resistance syndrome. Hepatology 2002;35:373-379.

27. Bugianesi E, Leone N, Vanni E, Marchesini G, Brunello F, Carucci P, et al. Expanding the natural history of nonalcoholic steatohepatitis: from cryptogenic cirrhosis to hepatocellular carcinoma. Gastroenterology 2002;123:134-140. 
28. Scaglioni F, Ciccia S, Marino M, Bedogni G, Bellentani S. ASH and NASH. Dig Dis 2011;29:202-210.

29. Brunt EM. Histopathology of non-alcoholic fatty liver disease. Clin Liver Dis 2009;13:533-544.

30. Zhang DY, Friedman SL. Fibrosis-dependent mechanisms of hepatocarcinogenesis. Hepatology 2012;56:769-775.

31. Sidharthan S, Kottilil S. Mechanisms of alcohol-induced hepatocellular carcinoma. Hepatol Int 2013;8:452-457.

32. Stickel F, Schuppan D, Hahn EG, Seitz HK. Cocarcinogenic effects of alcohol in hepatocarcinogenesis. Gut 2002;51:132-139.

33. Michelotti GA, Mariana MV, Diehl AM. NAFLD, NASH and liver cancer. Nat Rev Gastroenterol Hepatol 2013;10:656-665.

34. Caldwell SH, Oelsner DH, lezzoni JC, Hespenheide EE, Battle EH, Driscoll CJ. Cryptogenic cirrhosis: clinical characterization and risk factors for underlying disease. Hepatology 1999;29:664-669.

35. Starley BQ, Calcagno CJ, Harrison SA. Nonalcoholic fatty liver disease and hepatocellular carcinoma: a weighty connection. Hepatology 2010;51:1820-1832.

36. Ascha MS, Hanouneh IA, Lopez R, Tamimi TA, Feldstein AF, Zein NN. The incidence and risk factors of hepatocellular carcinoma in patients with nonalcoholic steatohepatitis. Hepatology 2010;51:19721978.

37. Shimizu I, Kamochi M, Yoshikawa H, Nakayama N. Gender difference in alcoholic liver disease. In: Shimizu I, ed. Trends in alcoholic liver disease research: clinical and scientific aspects. Rijeka: InTech, 2012:23-40.

38. Wilsnack RW, Wilsnack SC, Kristjanson AF, Vogeltanz-Holm ND, Gmel G. Gender and alcohol consumption: pattern from the multinational GENACIS project. Addiction 2009;104:1487-1500.

39. French DJ, Sargent-Cox KA, Kim S, Anstey KJ. Gender differences in alcohol consumption among middle-aged and older adults in Australia, the United States and Korea. Aust N Z J Public Health 2014;38:332-339.

40. Tokushige K, Hashimoto E, Horie Y, Taniai M, Higuchi S. Hepatocellular carcinoma in Japanese patients with nonalcoholic fatty liver disease, alcoholic liver disease, and chronic liver disease of unknown etiology: report of the nationwide survey. J Gastroenterol 2011;46:1230-1237.
41. Yasui K, Hashimoto E, Komorizono Y, Koike K, Arii S, Imai Y, et al. Characteristics of patients with nonalcoholic steatohepatitis who develop hepatocellular carcinoma. Clin Gastroenterol Hepatol 2011;9:428-433; quiz e50.

42. Stine JG, Wentworth BJ, Zimmet A, Rinella ME, Loomba R, Caldwell $\mathrm{SH}$, et al. Systemic review with meta-analysis: risk of hepatocellular carcinoma in non-alcoholic steatohepatitis without cirrhosis compared to other liver diseases. Aliment Pharmacol Ther 2018;48:696703.

43. Mittal S, El-Serag HB, Sada YH, Kanwal F, Duan Z, Temple S, et al. Hepatocellular carcinoma in the absence of cirrhosis in United States veterans is associated with nonalcoholic fatty liver disease. Clin Gastroenterol Hepatol 2016;14:124-131.e1.

44. Seitz HK, Stickel F. Risk factors and mechanisms of hepatocarcinogenesis with special emphasis on alcohol and oxidative stress. Biol Chem 2006;387:349-360.

45. Bralet MP, Régimbeau JM, Pineau P, Dubois S, Loas G, Degos F, et al. Hepatocellular carcinoma occurring in nonfibrotic liver: epidemiologic and histopathologic analysis of 80 French cases. Hepatology 2000;32:200-204.

46. Bruix J, Sherman M; American Association for the Study of Liver Diseases. Management of hepatocellular carcinoma: an update. Hepatology 2011;53:1020-1022.

47. Kanwal F, Kramer JR, Mapakshi S, Natarajan Y, Chayanupatkul M, Richardson PA, et al. Risk of hepatocellular cancer in patient with non-alcoholic fatty liver disease. Gastroenterology 2018;155:1828 1837.e2.

48. Flemming JA, Yang JD, Vittinghoff E, Kim WR, Terrault NA. Risk prediction of hepatocellular carcinoma in patients with cirrhosis: the ADRESS-HCC risk model. Cancer 2014;120:3485-3493.

49. Weinmann A, Alt Y, Koch S, Nelles C, Düber C, Lang H, et al. Treatment and survival of non-alcoholic steatohepatitis associated hepatocellular carcinoma. BMC Cancer 2015;15:210.

50. Costentin CE, Mourad A, Lahmek $P$, Causse X, Pariente A, Hagège $H$, et al. Hepatocellular carcinoma is diagnosed at a later stage in alcoholic patients: results of a prospective, nationwide study. Cancer 2018;124:1964-1972. 